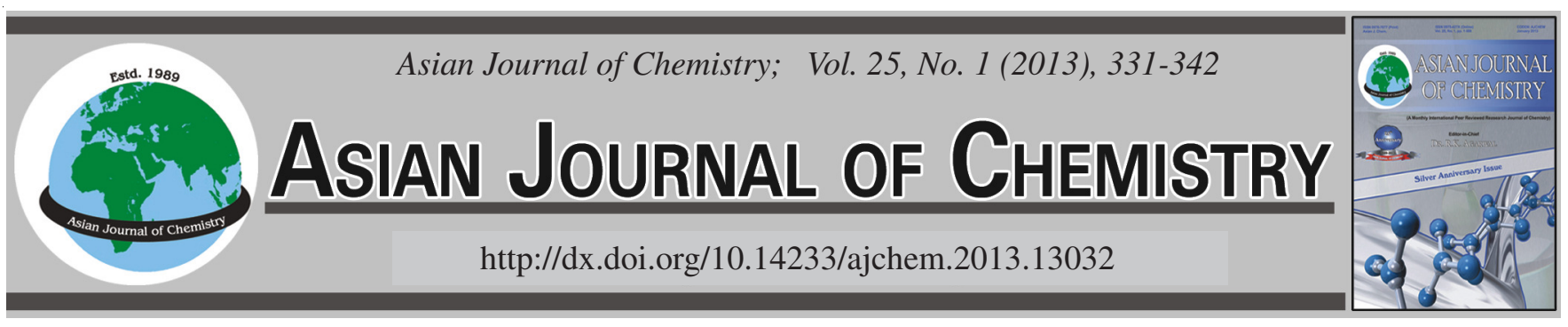

\title{
Chemometric Modeling to Predict Aquatic Toxicity of Benzene Derivatives Using Stepwise-Multi Linear Regression and Partial Least Square
}

\author{
Maryam Bordbar ${ }^{1, *}$, Jahanbakhsh Ghasemi $^{2}$, Ali $_{\text {Yeganeh }}$ FaAl $^{3}$ and Razieh Fazaeli ${ }^{4}$
}

\author{
${ }^{1}$ Department of Chemistry, Faculty of Science, University of Qom, P.O. Box 37185-359, Qom, Iran \\ ${ }^{2}$ Department of Chemistry, K.N. Toosi University of Technology, P.O. Box 16167-45481, Tehran, Iran \\ ${ }^{3}$ Department of Chemistry, Payame Noor University, P.O. Box 19395-3697, Iran \\ ${ }^{4}$ Department of Chemistry, Shahreza Branch, Islamic Azad University, P.O. Box 86145-311, Shreza, Iran
}

*Corresponding author: Fax: +98 251 2916449; Tel: +98 251 2853311; E-mail: m.bordbar@qom.ac.ir

\begin{abstract}
The aquatic toxicity of 392 benzene derivatives have been subjected to quantitative structure-activity relationship studies. Optimization of 3D structures of the molecules carried out by HyperChem using AM1 model. The molecular descriptors; constitutional, topological, molecular walk counts, aromaticity indices, geometrical, WHIM, functional group, empirical and properties were obtained by Dragon software. The models were constructed using 309 molecules as training set and predictive ability tested using 78 compounds. Modeling of $\log \left(1 / \mathrm{IGC}_{50}\right)$ of these compounds as a function of the theoretically derived descriptors was established by multiple linear regression (MLR) technique. This linear modeling method indicates the importance of different topological and electronic descriptors on the aquatic toxicity $\left[\log \left(1 / \mathrm{IGC}_{50}\right)\right]$. The obtained model (stepwise MLR-PLS) was chosen based on highest external predictive $\mathrm{R}^{2}$ value $(0.81)$ and lowest RMSEP (2.41) values. It is observed that the Moriguchi octanol/water partition coefficient $(\log \mathrm{P})$ descriptor has great effect on the aquatic toxicity, which confirms its importance in mechanism of aquatic toxicity action of benzene derivatives.
\end{abstract}

Key Words: Quantitative structure-activity relationship, Multiple linear regression, Partial least square, Aquatic toxicity, Benzene derivatives.

\section{INTRODUCTION}

Structure-toxicity models exist at the intersection of biology, chemistry and statistics. The connection of these three subjects has permitted the development of structure-activity relationships as an accepted sub-discipline in toxicology. The next decade will see an increased use of (quantitative) structureactivity relationships (QSARs) to predict toxicity for new and existing chemicals. Much of the focus will be on their application to reduce or replace animal use in toxicological testing for the regulation of existing chemicals (e.g. in the REACH legislation $)^{1}$. The official birth date of QSAR is considered to be 1962, when Hansch et al. ${ }^{2}$ published a paper which showed a correlation between biological activity and octanol-water partition coefficient. Quantitative structure-activity relationship models have another ability which is obtaining a deeper knowledge about the mechanism of biological activity. Quantitative structure-activity relationships represent predictive models derived from application of statistical tools correlating biological activity (including therapeutic and toxic) of chemicals (drugs/toxicants/environmental pollutants) with descriptors representative of molecular structure and/or property. The concept that there exists a close relationship between bulk properties of similar compounds and their molecular structure allows one to provide a clear connection between the macroscopic and the microscopic properties of matter. Quantitative structure-property relationships are mathematical equations relating chemical structure to a wide variety of physical, chemical, biological and technological properties.

Nowadays, a wide range of descriptors has been used in QSAR modeling ${ }^{3}$. These descriptors have been classified into different categories according to Karelson approach including constitutional, geometrical, topological and quantum chemical, etc. ${ }^{4}$.

The success of any QSAR model depends on the accuracy of input data, selection of appropriate descriptors that represent variations in structural property of molecules quantitatively and statistical tools and validation of the developed model ${ }^{5}$. The validation strategies check the reliability of the developed models for their possible application on a new set of data and confidence of prediction can thus be judged. For validation of QSAR models usually four strategies are adopted ${ }^{6}$ : (a) Internal validation or cross-validation; (b) Validation by dividing the 
data set into training and test compounds; (c) True external validation by application of model on external data and (d) Data randomization or Y-scrambling. As a result, a simple mathematical relationship is established:

Property $=\mathrm{f}$ (Structural descriptors)

Quantitative structure-activity relationship techniques include from chemical measurements and biological assays to the statistical techniques and interpretation of results ${ }^{7}$.

In this work a QSAR study is performed; to develop model that relate the structures of 392 substituted benzenes to toxic action. The stepwise multiple linear regression were used to select the most informative descriptors from the calculated descriptors by Dragon (version 2.1) software ${ }^{3}$. The selected descriptors were used to develop a model for predicting the $\log \left(\mathrm{IGC}_{50}\right)$ (decimal logarithm of the inverse $50 \%$ growth inhibitory concentration) values. We have validated the models by dividing the data set into training (307 compounds) and test set (78 compounds) by K-means clustering technique. Different statistical techniques were used to develop the model to highlight the structural requirements for an ideal aquatic toxicity inhibitor. The two objectives of the present paper have been: (1) To explore the structure-activity relationships of aquatic toxicity of diverse compounds and (2) To select the best predictive model from among all comparable chemometric models for the aquatic toxicity.

\section{EXPERIMENTAL}

The QSAR model for the estimation of the $\log \left(\mathrm{IGC}_{50}\right)$ of various substituted benzenes compounds is established in the following five steps: (i) the molecular structure input and generation of the files containing the chemical structures is stored in a computer-readable format; (ii) quantum mechanics geometry is optimized with a semi-empirical (AM1) method; (iii) structural descriptors are computed; (iv) structural descriptors are selected by stepwise multiple linear regression; (v) the structure$\log \left(\mathrm{IGC}_{50}\right)$ model is generated by partial least square calibration method and statistical analysis.

Chemical dataset selection: Central to the issues of quality, transparency and domain identification as they relate to toxicological QSAR is biological data. High-quality toxicity data, in a structurally diverse set of molecules are required to formulate and validate high-quality QSARs. Quality toxicity data typically come from standardized assays measured in a consistent manner, with a clear and unambiguous end point and lower experimental error ${ }^{8}$. Toxicity assessments which are made in a single laboratory by a single protocol tend to be the most precise. By taking these points into consideration, we select the database of inhibition of growth of the ciliated protozoan T. pyriformis. This database has been developed in a single laboratory over more than two decades and it has been recognized as a high-quality dataset ${ }^{9}$.

The general dataset used in this study has been recently published by other researchers ${ }^{8}$. It consists of almost 400 substituted benzenes representing several mechanisms of toxic action (Table-1). Some compounds were reported by Schultz and Netzeva as not toxic at saturation. Hence these compounds were not used in the present work. A horizontal validation was performed using a training set, composed by 307 benzene derivatives, for models development and a validation set (78 compounds) to assess the predictive capability of the QSAR models. In order to split the database into training and prediction series, a k-means cluster analyses (k-MCA) was carried out for entire dataset to design, in a rational representative way, the training (training) and prediction (test) series ${ }^{10}$.

Computer hardware and software: All calculations were run on a Pentium IV personal computer with windows XP operating system. The ChemDrawUltra version 9.0 (Chem Office 2005, CambridgeSoft Corporation) software was used for drawing the molecular structures. The optimizations of molecular structures were done by the HyperChem 7.5 (AM1 method) and descriptors were calculated by Dragon (Milano Chemometrics group, version 2.1) software. Stepwise multiple linear regression regression was performed by using SPSS version 11.5 software and partial least square calculations were performed in the MATLAB (version 7.5, MathWorks Inc.).

Molecular modeling and theoretical molecular descriptors: Molecular descriptors define the molecular structure and physicochemical properties of molecules by a single number. A wide variety of descriptors have been reported for using in QSAR analysis ${ }^{3,11}$. The structures were drawn in Chem Draw Ultra version 9.0 and exported in a file format suitable for HyperChem 7.5. The geometry optimization was performed with the semi-empirical quantum method Austin Method 1 $(\mathrm{AM} 1)^{12}$ incorporated in the HyperChem program. The gradient norm criterion $0.01 \mathrm{kcal} / \mathrm{A}^{\circ}$ was applied in the geometry optimization for all structures. The HyperChem mol files were used by the Dragon program to compute more than 1027 structural descriptors for the 392 benzene derivatives. Dragon computes 10 classes of structural descriptors: constitutional (number of various types of atoms and bonds, number of rings, molecular weight, etc.); topological (Wiener index, Randic indices, Kier-Hall shape indices, Balaban index, etc.); geometrical (moments of inertia, molecular volume, molecular surface area, etc.); electrostatic (minimum and maximum partial charges, polarity parameter, charged partial surface area descriptors, etc.); Molecular walk counts (molecular walk counts of order 1-10, total walk count, self-returning of order 1-10); aromaticity indices (harmonic oscillator model of aromaticity index, Jug RC index, aromaticity, HOMA total); WHIM descriptors (unweighted size, shape, symmetry and accessibility directional indices; size, shape, symmetry and accessibility directional indices weighted by atomic polarizability, atomic Sanderson electronegativity or atomic van der Waals volume; total size, shape symmetry and accessibility indices); functional group (numbers of different types of carbons, number of allenes groups, number of esters (aliphatic or aromatic), number of amides, number of different functional groups, number of $\mathrm{CH} 3 \mathrm{R}$, number of $\mathrm{CR} 4$, number of different halogens attached to different type of carbons, number of PX3, number of PR3); empirical (Unsaturation index, ydrophilic factor, aromatic ratio) and properties descriptors (molar refractivity, polar surface area, $\log \mathrm{P}$ ) were generated for each compound.

The generation of the descriptors is carried out without taking into account of the solvation of the molecules. It means that the generated descriptors are carried out using the 
TABLE-1

EXPERIMENTAL AND PREDICTED VALUES $\left[\log \left(1 / \mathrm{IGC}_{50}\right)\right]$ FOR THE TRAINING AND TEST SET BY STEPWISE MLR-PLS MODEL

\begin{tabular}{|c|c|c|c|c|c|}
\hline \multicolumn{2}{|c|}{ Compounds } & \multirow{2}{*}{$\frac{\text { CAS }}{71-43-2}$} & \multirow{2}{*}{$\frac{\log \left(1 / \mathrm{IGC}_{50}\right)}{-0.12}$} & \multirow{2}{*}{$\begin{array}{r}\text { Training set } \\
-0.09\end{array}$} & \multirow[t]{2}{*}{ Test set } \\
\hline 001 & Benzene & & & & \\
\hline 002 & $p$-Xylene & $106-42-3$ & 0.25 & 0.37 & \\
\hline 003 & 1-Phenyl-2-butanol & $120055-09-6$ & -0.16 & 0.14 & \\
\hline 004 & Toluene & $108-88-3$ & 0.25 & -0.04 & \\
\hline 005 & $n$-Butylbenzene & $104-51-8$ & 1.25 & 0.82 & \\
\hline 006 & $n$-Amylbenzene & $538-68-1$ & 1.79 & 1.19 & \\
\hline 007 & Benzylamine & $100-46-9$ & -0.24 & -0.68 & \\
\hline 008 & Isopropylbenzene & $98-82-8$ & 0.69 & 0.45 & \\
\hline 009 & 6-Phenyl-1-hexanol & 2430-16-2 & 0.87 & 0.95 & \\
\hline 010 & 5-Phenyl-1-pentanol & $10521-91-2$ & 0.42 & & 0.64 \\
\hline 011 & $\alpha, \alpha$-Ethylbenzenepropanol & $103-05-9$ & -0.07 & -0.09 & \\
\hline 012 & 4-Phenyl-1-butanol & $3360-41-6$ & 0.12 & 0.23 & \\
\hline 013 & 3-Phenyl-1-propanol & $122-97-4$ & -0.21 & -0.11 & \\
\hline 014 & Benzyl alcohol & $100-51-6$ & -0.83 & & -0.41 \\
\hline 015 & sec-Phenethyl alcohol & $98-85-1$ & -0.66 & -0.46 & \\
\hline 016 & 4-Ethylbenzyl alcohol & $768-59-2$ & 0.07 & 0.10 & \\
\hline 017 & 3-Phenyl-1-butanol & $2722-36-3$ & 0.01 & & 0.14 \\
\hline 018 & (R)-1-phenyl-1-butanol & 22144-60-1 & -0.01 & 0.08 & \\
\hline 019 & 4-Biphenylmethanol & $3597-91-9$ & 0.92 & & 0.66 \\
\hline 020 & 4-Ethylbiphenyla & $5707-44-8$ & 1.97 & 1.44 & \\
\hline 021 & Biphenyl & $92-52-4$ & 1.05 & 1.11 & \\
\hline 022 & ( \pm )-2-Phenyl-2-butanol & $1565-75-9$ & 0.06 & -0.31 & \\
\hline 023 & ( \pm -1,2-Diphenyl-2-propanol & $5342-87-0$ & 0.80 & 0.62 & \\
\hline 024 & 1,1-Diphenyl-2-propanol & $29338-49-6$ & 0.75 & & 0.66 \\
\hline 025 & 3,4-Dimethylaniline & $95-64-7$ & -0.16 & 0.19 & \\
\hline 026 & 3-Aminobenzyl alcohol & $1877-77-6$ & -1.13 & -0.03 & \\
\hline 027 & 4-Butoxyaniline & $4344-55-2$ & 0.61 & 0.67 & \\
\hline 028 & 4-Pentyloxyaniline & $39905-50-5$ & 0.97 & & 0.99 \\
\hline 029 & 4-Hexyloxyaniline & $39905-57-2$ & 1.38 & 1.29 & \\
\hline 030 & 4-Methylaniline & $106-49-0$ & -0.05 & -0.13 & \\
\hline 031 & 4-Isopropylaniline & $99-88-7$ & 0.22 & 0.12 & \\
\hline 032 & 3-Ethylaniline & $587-02-0$ & -0.03 & 0.00 & \\
\hline 033 & 4-Ethylaniline & $589-16-2$ & 0.03 & -0.07 & \\
\hline 034 & 3-Methylaniline & $108-44-1$ & 0.28 & -0.18 & \\
\hline 035 & 4-Butylaniline & $104-13-2$ & 1.07 & 0.60 & \\
\hline 036 & (2-Bromoethyl)benzene & $103-63-9$ & 0.42 & 0.01 & \\
\hline 037 & 2-Methylaniline & $95-53-4$ & -0.16 & & -0.21 \\
\hline 038 & 2,6-Diisopropylaniline & $24544-04-5$ & 0.76 & 0.99 & \\
\hline 039 & Aniline & $62-53-3$ & -0.23 & & -0.24 \\
\hline 040 & 2-Ethylaniline & $578-54-1$ & -0.22 & -0.16 & \\
\hline 041 & 2,6-Diethylaniline & $579-66-8$ & 0.31 & 0.45 & \\
\hline 042 & Thioanisole & $100-68-5$ & 0.18 & 0.13 & \\
\hline 043 & 4-Methoxyphenol & $150-76-5$ & -0.14 & & -0.18 \\
\hline 044 & 3,4,5-Trimethylphenol & $527-54-8$ & 0.93 & & 0.77 \\
\hline 045 & Benzyl chloride & $100-44-7$ & 0.06 & 0.07 & \\
\hline 046 & 4-Methylanisole & $104-93-8$ & 0.25 & 0.14 & \\
\hline 047 & 2,3,5-Trimethylphenol & $697-82-5$ & 0.36 & 0.68 & \\
\hline 048 & 2,4,6-Trimethylphenol & $527-60-6$ & 0.42 & 0.56 & \\
\hline 049 & 4-tert-Butylpheno & $98-54-4$ & 0.91 & 0.43 & \\
\hline 050 & 4-tert-Pentylphenol & $80-46-6$ & 1.23 & & 0.63 \\
\hline 051 & 2,3,6-Trimethylphenol & $2416-94-6$ & 0.28 & 0.54 & \\
\hline 052 & Phenetole & $103-73-1$ & -0.14 & 0.14 & \\
\hline 053 & Anisole & $100-66-3$ & -0.10 & & -0.21 \\
\hline 054 & 2,4-Dimethylphenol & $105-67-9$ & 0.14 & 0.34 & \\
\hline 055 & 2-Phenyl-3-butyn-2-ol & $127-66-2$ & -0.18 & -0.24 & \\
\hline 056 & $p$-Cresol & $106-44-5$ & -0.16 & 0.00 & \\
\hline 057 & 4-Ethylphenol & $123-07-9$ & 0.21 & 0.09 & \\
\hline 058 & 4-Propylphenol & $645-56-7$ & 0.64 & 0.40 & \\
\hline 059 & 3-Ethylphenol & $620-17-7$ & 0.29 & 0.13 & \\
\hline 060 & Nonylphenol & $104-40-5$ & 2.47 & 2.59 & \\
\hline 061 & $m$-Cresol & $108-39-4$ & -0.08 & -0.04 & \\
\hline 062 & $o$-Cresol & $95-48-7$ & -0.29 & -0.06 & \\
\hline 063 & 2-Ethylphenol & $90-00-6$ & 0.16 & 0.00 & \\
\hline 064 & Phenol & $108-95-2$ & -0.35 & 0.03 & \\
\hline
\end{tabular}




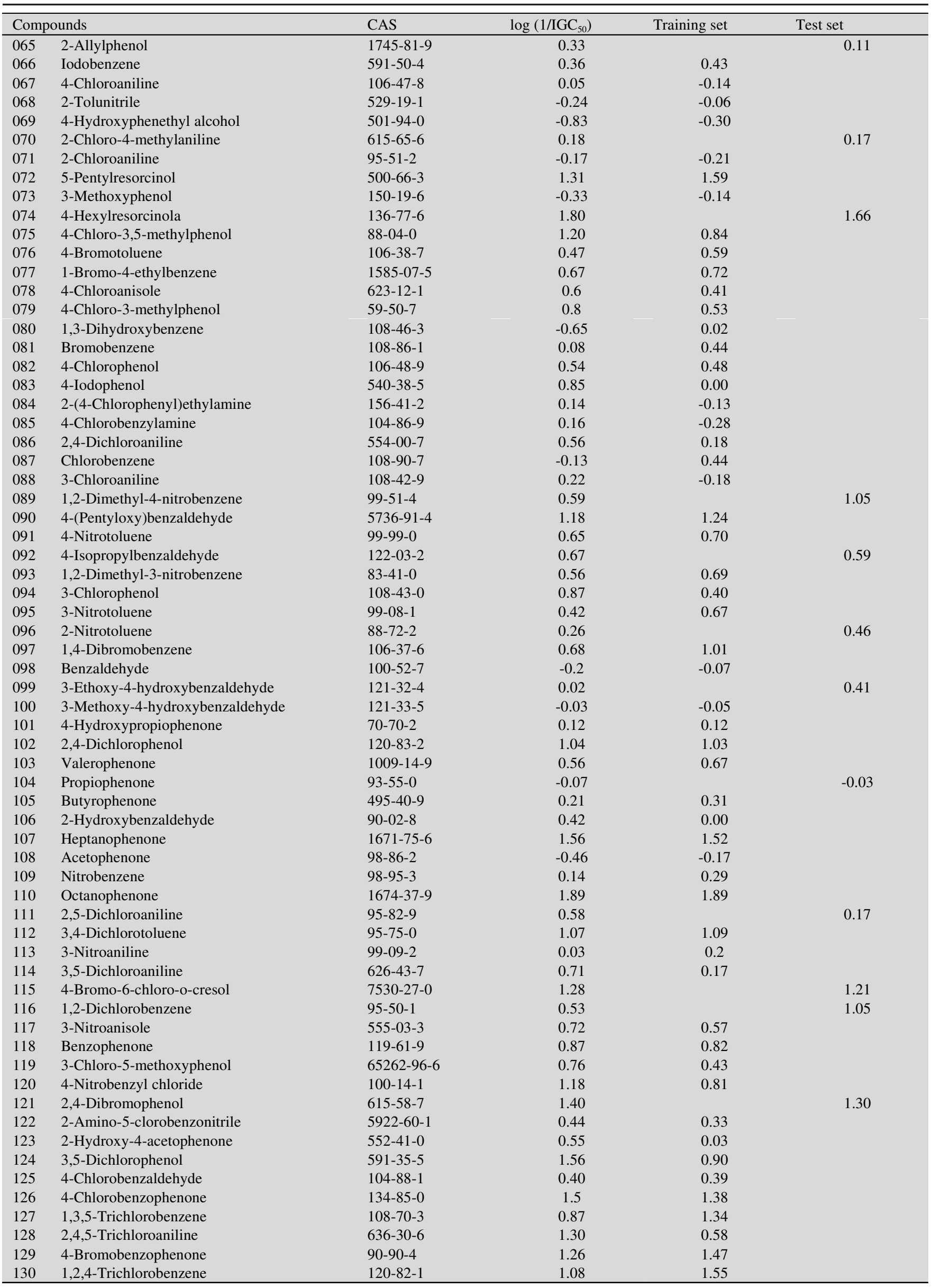




\begin{tabular}{|c|c|c|c|c|c|}
\hline \multicolumn{2}{|c|}{ Compounds } & \multirow{2}{*}{$\begin{array}{l}\text { CAS } \\
88-06-2\end{array}$} & \multirow{2}{*}{$\frac{\log \left(1 / \mathrm{IGC}_{50}\right)}{1.41}$} & \multirow{2}{*}{$\begin{array}{c}\text { Training set } \\
1.37\end{array}$} & \multirow[t]{2}{*}{ Test set } \\
\hline 131 & 2,4,6-Trichlorophenol & & & & \\
\hline 132 & 4-Ethoxy-2-nitroaniline & $616-86-4$ & 0.76 & 1.03 & \\
\hline 133 & 5-Bromovanillin & $2973-76-4$ & 0.62 & 0.48 & \\
\hline 134 & 4-Nitrophenetole & $100-29-8$ & 0.83 & 0.84 & \\
\hline 135 & 4-Chloro-2-nitrotoluene & $89-59-8$ & 0.82 & 1.15 & \\
\hline 136 & 1-Bromo-3-nitrobenzene & $585-79-5$ & 1.03 & 0.96 & \\
\hline 137 & 4-Bromo-2,6-dichlorophenol & $3217-15-0$ & 1.78 & 1.80 & \\
\hline 138 & 2-Chloro-6-nitrotoluene & $83-42-1$ & 0.68 & 1.00 & \\
\hline 139 & 2,3,5,6-Tetrachloroaniline & $3481-20-7$ & 1.76 & & 0.95 \\
\hline 140 & 3-Nitrobenzonitrile & $619-24-9$ & 0.45 & 0.38 & \\
\hline 141 & 2,4,5-Trichlorophenol & $95-95-4$ & 2.10 & 1.60 & \\
\hline 142 & 1,2,4,5-Tetrachlorobenzene & 95-94-3 & 2.00 & 2.05 & \\
\hline 143 & 4-Methyl-2-nitroaniline & $89-62-3$ & 0.37 & 0.42 & \\
\hline 144 & 1-Chloro-3-nitrobenzene & $121-73-3$ & 0.73 & 0.89 & \\
\hline 145 & 2-Nitroaniline & $88-74-4$ & 0.08 & -0.03 & \\
\hline 146 & 2,3,4,5-Tetrachloroaniline & $634-83-3$ & 1.96 & 1.04 & \\
\hline 147 & 2,4,6-Tribromophenol & $118-79-6$ & 1.91 & 2.03 & \\
\hline 148 & 2-Bromo-5-nitrotoluene & $7149-70-4$ & 1.16 & 1.50 & \\
\hline 149 & 1-Fluoro-3-iodo-5-nitrobenzene & $3819-88-3$ & 1.09 & & 1.09 \\
\hline 150 & 2-Nitrophenol & $88-75-5$ & 0.67 & 0.20 & \\
\hline 151 & 2-Chloro-4-nitroaniline & $121-87-9$ & 0.75 & 0.74 & \\
\hline 152 & 5-Hydroxy-2-nitrobenzaldehyde & $42454-06-8$ & 0.33 & 0.28 & \\
\hline 153 & 3,4,5,6-Tetrabromo-o-cresol & $576-55-6$ & 2.57 & $*$ & \\
\hline 154 & 2,3,4,6-Tetrachlorophenol & $58-90-2$ & 2.18 & 1.91 & \\
\hline 155 & 1-Fluoro-4-nitrobenzene & $350-46-9$ & 0.1 & 0.71 & \\
\hline 156 & Pentafluoro aniline & $771-60-8$ & 0.26 & 0.66 & \\
\hline 157 & 1-Bromo-2-nitrobenzene & $577-19-5$ & 0.75 & 0.60 & \\
\hline 158 & 3,5-Dibromo-salicylaldehyde & $90-59-5$ & 1.65 & 1.29 & \\
\hline 159 & 3,5-Dichloro-nitrobenzene & $618-62-2$ & 1.13 & 1.48 & \\
\hline 160 & 4-Chloro-3-nitrophenol & $610-78-6$ & 1.27 & & 0.89 \\
\hline 161 & $2,3,4,5$-Tetrachlorophenol & $4901-51-3$ & 2.72 & 2.02 & \\
\hline 162 & Thiobenzamide & $2227-79-4$ & 0.09 & & -0.04 \\
\hline 163 & 1-Chloro-4-nitrobenzene & $100-00-5$ & 0.43 & 0.81 & \\
\hline 164 & $4,4,4,4$-Tetrafluoro- $m$-toluidine & $2357-47-3$ & 0.77 & 0.59 & \\
\hline 165 & 1-Chloro-2-nitrobenzene & $88-73-3$ & 0.68 & 0.76 & \\
\hline 166 & 4-Chloro-6-nitro- $m$-cresol & $7147-89-9$ & 1.63 & 1.09 & \\
\hline 167 & Pentachlorophenol & $87-86-5$ & 2.07 & 2.33 & \\
\hline 168 & 1,3-Dinitrobenzene & $99-65-0$ & 0.76 & 1.07 & \\
\hline 169 & 2,4-Dinitrotoluene & $121-14-2$ & 0.87 & 1.39 & \\
\hline 170 & 4,5-Dichloro-2-nitroaniline & 6641-64-1 & 1.66 & 1.36 & \\
\hline 171 & Pentafluorophenol & $771-61-9$ & 1.63 & 1.58 & \\
\hline 172 & Pentabromophenol & $608-71-9$ & 2.66 & $*$ & \\
\hline 173 & 3-Chloro-4-fluoronitrobenzene & $350-30-1$ & 0.8 & 1.20 & \\
\hline 174 & 1,4-Dinitrobenzene & $100-25-4$ & 1.30 & 1.05 & \\
\hline 175 & 3,4-Dichloronitrobenzene & $99-54-7$ & 1.16 & 1.44 & \\
\hline 176 & 2,5-Dichloronitrobenzene & $89-61-2$ & 1.13 & 1.38 & \\
\hline 177 & 2,4-Dichloro-6-nitroaniline & $2683-43-4$ & 1.26 & 1.17 & \\
\hline 178 & 3,4-Dinitrobenzyl alcohol & $79544-31-3$ & 1.09 & 0.74 & \\
\hline 179 & 2,4-Dichloronitrobenzene & $611-06-3$ & 0.99 & & 1.25 \\
\hline 180 & 2,3-Dichloronitrobenzene & $3209-22-1$ & 1.07 & 1.06 & \\
\hline 181 & 1,2-Dinitrobenzene & $528-29-0$ & 1.25 & 0.69 & \\
\hline 182 & Phenyl isothiocyanate & $103-72-0$ & 1.41 & 0.25 & \\
\hline 183 & 3-Trifluoromethyl-4-ntrophenol & $88-30-2$ & 1.65 & 1.19 & \\
\hline 184 & 2,6-Iodo-4-nitrophenol & $305-85-1$ & 1.81 & & 1.01 \\
\hline 185 & 2,4-Chloro-6-nitrophenol & $609-89-2$ & 1.75 & 1.29 & \\
\hline 186 & 1,3,5-Trichloro-2-nitrobenzene & $18708-70-8$ & 1.43 & 1.52 & \\
\hline 187 & 1,2,4-Trichloro-5-nitrobenzene & $89-69-0$ & 1.53 & 1.93 & \\
\hline 188 & 1,2,3-Trichloro-4-nitrobenzene & $17700-09-3$ & 1.51 & 1.72 & \\
\hline 189 & 2-Chloro-5-nitrobenzaldehyde & $6361-21-3$ & 0.53 & 0.94 & \\
\hline 190 & Pentafluorobenzaldehyde & $653-37-2$ & 0.82 & 1.06 & \\
\hline 191 & 2,4-Dinitro-1-iodobenzene & $709-49-9$ & 2.12 & & 1.90 \\
\hline 192 & 2,3,5,6-Tetrachloronitrobenzene & $117-18-0$ & 1.82 & & 2.00 \\
\hline 193 & 2,5-Dinitrophenol & $329-71-5$ & 1.04 & 0.95 & \\
\hline 194 & 2,4-Dinitroaniline & $97-02-9$ & 0.72 & 0.73 & \\
\hline 195 & 2,3,4,5-Tetrachloronitrobenzene & $879-39-0$ & 1.78 & 2.30 & \\
\hline 196 & 1,2,3-Trifluoro-4-nitrobenzene & $771-69-7$ & 1.89 & 1.25 & \\
\hline
\end{tabular}




\begin{tabular}{|c|c|c|c|c|c|}
\hline \multicolumn{2}{|c|}{ Compounds } & \multirow{2}{*}{$\frac{\text { CAS }}{6306-39-4}$} & \multirow{2}{*}{$\frac{\log \left(1 / \mathrm{IGC}_{50}\right)}{2.21}$} & \multirow{2}{*}{$\begin{array}{c}\text { Training set } \\
2.17\end{array}$} & \multirow[t]{2}{*}{ Test set } \\
\hline 197 & 1,2-Dichloro-4,5-dinitrobenzene & & & & \\
\hline 198 & 2,6-Dinitroaniline & $606-22-4$ & 0.84 & 0.62 & \\
\hline 199 & 4,6-Dinitro-2-methylphenol & $534-52-1$ & 1.73 & & 1.18 \\
\hline 200 & 4-tert-Butyl-2,6-dinitrophenol & $4097-49-8$ & 1.8 & 1.97 & \\
\hline 201 & 1-Bromo-2,4-dinitrobenzene & $584-48-5$ & 2.31 & 1.54 & \\
\hline 202 & 2,4-Dinitrophenol & $51-28-5$ & 1.06 & 0.99 & \\
\hline 203 & 1,5-Dichloro-2,3-dinitrobenzene & 28689-08-9 & 2.42 & 1.98 & \\
\hline 204 & 6-Chloro-2,4-dinitroaniline & $3531-19-9$ & 1.12 & & 1.36 \\
\hline 205 & 2-Bromo-4,6-dinitroaniline & $1817-73-8$ & 1.24 & 1.61 & \\
\hline 206 & 2,3,4,6-tetrafluoronitrobenzene & $314-41-0$ & 1.87 & 1.23 & \\
\hline 207 & 2,6-Dinitrophenol & $573-56-8$ & 0.83 & & 0.67 \\
\hline 208 & 1-Chloro-2,4-dinitrobenzene & $97-00-7$ & 2.16 & & 1.54 \\
\hline 209 & 2,4-Dinitro-1-fluorobenzene & $70-34-8$ & 1.71 & 1.34 & \\
\hline 210 & Pentafluoronitrobenzene & $880-78-4$ & 2.43 & & 2.15 \\
\hline 211 & 1,4-dinitrotetrachlorobenzene & $20098-38-8$ & 2.82 & 2.96 & \\
\hline 212 & 1,5-Difluoro-2,4-dinitrobenzene & $327-92-4$ & 2.08 & 1.57 & \\
\hline 213 & 1,3-Dinitro-2,4,5trichlorobenzene & $2678-21-9$ & 2.60 & 2.32 & \\
\hline 214 & 1,3,5-Trichloro-2,4dinitrobenzene hemihydrate & $6284-83-9$ & 2.19 & 2.2 & \\
\hline 215 & 4-Chloro-3,5-dinitrobenzaldehydea & $1930-72-9$ & 2.66 & 1.47 & \\
\hline 216 & 1-Phenyl-2-propanol & $14898-87-4$ & -0.62 & -0.26 & \\
\hline 217 & 4-Methylbenzyl alcohol & $589-18-4$ & -0.49 & -0.07 & \\
\hline 218 & $( \pm) 1-P h e n y l-2-$ pentanol & $705-73-7$ & 0.16 & 0.45 & \\
\hline 219 & 4-Isopropylbenzyl alcohol & $536-60-7$ & 0.18 & & 0.39 \\
\hline 220 & 2 -(p-Tolyl)ethylamine & $3261-62-9$ & -0.04 & -0.12 & \\
\hline 221 & 4-Methyl benzylamine & $104-84-7$ & -0.01 & -0.28 & \\
\hline 222 & 3-Methylbenzyl alcohol & $587-03-1$ & -0.24 & -0.08 & \\
\hline 223 & 3-Phenyl-2-propen-1-ol & $104-54-1$ & -0.08 & & 0.06 \\
\hline 224 & 4-tert-Buthylbenzyl alcohol & $877-65-6$ & 0.48 & 0.47 & \\
\hline 225 & 4-Methylphenetyl alcohol & $699-02-5$ & -0.26 & 0.01 & \\
\hline 226 & 1-Phenylethylamine & $618-36-0$ & -0.18 & & -0.53 \\
\hline 227 & 2-Methylbenzyl alcohol & $89-95-2$ & -0.43 & & -0.39 \\
\hline 228 & 2-Methyl-1-phenyl-2-propanol & $100-86-7$ & -0.41 & -0.5 & \\
\hline 229 & $N$-Methylphenethylamine & $589-08-2$ & -0.41 & -0.27 & \\
\hline 230 & b-Methylphenethylamine & $582-22-9$ & -0.28 & -0.17 & \\
\hline 231 & $( \pm)$-1-Phenyl-1-butanol & $22135-49-5$ & -0.09 & 0.08 & \\
\hline 232 & $( \pm)$-1-Phenyl-1-propanol & $93-54-9$ & -0.43 & -0.28 & \\
\hline 233 & Phenetyl alcohol & $60-12-8$ & -0.59 & -0.37 & \\
\hline 234 & 2-Phenyl-1-propanol & $1123-85-9$ & -0.4 & -0.26 & \\
\hline 235 & 2-Phenyl-2-propanol & $617-94-7$ & -0.57 & -0.51 & \\
\hline 236 & 2-Phenyl-1-butanol & 89104-46-1 & -0.11 & & 0.11 \\
\hline 237 & Benzhydrol & $91-01-0$ & 0.5 & 0.41 & \\
\hline 238 & Benzaldoxime & $622-32-2$ & -0.11 & 0.07 & \\
\hline 239 & 3,5-Dimethylaniline & $108-69-0$ & -0.36 & 0.17 & \\
\hline 240 & 4-tert-Buthylaniline & $769-92-6$ & 0.36 & 0.25 & \\
\hline 241 & 2,4-Dimethylaniline & $95-68-1$ & -0.29 & 0.18 & \\
\hline 242 & 4-Phenylbutyronitrile & $2046-18-6$ & 0.15 & 0.31 & \\
\hline 243 & 2,4,6-Trimethylaniline & $88-05-1$ & -0.05 & 0.44 & \\
\hline 244 & 3-Phenylpropionitrile & $645-59-0$ & -0.16 & -0.01 & \\
\hline 245 & 4-sec-Butylaniline & $30273-11-1$ & 0.61 & 0.51 & \\
\hline 246 & 2,3-Dimethylaniline & $87-59-2$ & -0.43 & 0.14 & \\
\hline 247 & Benzyl cyanide & $140-29-4$ & -0.36 & -0.21 & \\
\hline 248 & 2,5-Dimethylaniline & $95-78-3$ & -0.33 & & 0.17 \\
\hline 249 & $\alpha$-Methylbenzyl cyanide & $1823-91-2$ & 0.01 & & 0.00 \\
\hline 250 & 2-Isopropylaniline & $643-28-7$ & 0.12 & 0.06 & \\
\hline 251 & 2,6-Dimethylaniline & $87-62-7$ & -0.43 & 0.03 & \\
\hline 252 & $N$-ethylaniline & $103-69-5$ & 0.07 & & -0.02 \\
\hline 253 & 2-Propylaniline & $1821-39-2$ & 0.08 & & 0.12 \\
\hline 254 & $N$-Methylaniline & $100-61-8$ & 0.06 & -0.31 & \\
\hline 255 & 2-Amino-4-tert-butylaniline & $1199-46-8$ & 0.37 & & 0.25 \\
\hline 256 & 2-Methoxyaniline & $90-04-0$ & -0.69 & -0.43 & \\
\hline 257 & 3-Phenylpyridine & $1008-88-4$ & 0.47 & & 0.58 \\
\hline 258 & 2-Aminobenzyl alcohol & $5344-90-1$ & -1.07 & -0.16 & \\
\hline 259 & 2-Benzylpyridine & $101-82-6$ & 0.38 & 0.52 & \\
\hline 260 & 3,5-Di-tert-butylphenol & $1138-52-9$ & 1.64 & 1.97 & \\
\hline 261 & Phenyl propargyl sulfide & $5651-88-7$ & 0.54 & 0.5 & \\
\hline 262 & 4-Ethoxyphenol & $622-62-8$ & 0.01 & & 0.16 \\
\hline
\end{tabular}




\begin{tabular}{|c|c|c|c|c|c|}
\hline \multicolumn{2}{|c|}{ Compounds } & \multirow{2}{*}{$\begin{array}{l}\text { CAS } \\
122-94-1\end{array}$} & \multirow{2}{*}{$\frac{\log \left(1 / \mathrm{IGC}_{50}\right)}{0.70}$} & \multirow{2}{*}{$\begin{array}{c}\text { Training set } \\
0.86\end{array}$} & \multirow[t]{2}{*}{ Test set } \\
\hline 263 & 4-Butoxyphenol & & & & \\
\hline 264 & 4-Benzylpyridine & $2116-65-6$ & 0.63 & & 0.66 \\
\hline 265 & 2-Phenylpyridine & $1008-89-5$ & 0.27 & 0.8 & \\
\hline 266 & 3,4-Dimethylphenol & $95-65-8$ & 0.12 & 0.36 & \\
\hline 267 & 3-tert-Buthylphenol & $585-34-2$ & 0.74 & 0.54 & \\
\hline 268 & 3,5-Dimethylphenol & $108-68-9$ & 0.11 & & 0.31 \\
\hline 269 & 6-tert-Buthyl-2,4-dimethylphenol & $1879-09-0$ & 1.16 & 0.98 & \\
\hline 270 & 4-Isopropylphenol & $99-89-8$ & 0.47 & 0.42 & \\
\hline 271 & 3-Isopropylphenol & $618-45-1$ & 0.61 & & 0.57 \\
\hline 272 & 2,3-Dimethylphenol & $526-75-0$ & 0.12 & 0.27 & \\
\hline 273 & 2,5-Dimethylphenol & $95-87-4$ & 0.14 & & 0.30 \\
\hline 274 & 4-Hydroxy-3-methoxybenzyl alcohol & $498-00-0$ & -0.7 & -0.19 & \\
\hline 275 & 2-Isopropylphenol & $88-69-7$ & 0.61 & 0.39 & \\
\hline 276 & 3-Amino-2-cresol & $53222-92-7$ & -0.55 & -0.02 & \\
\hline 277 & 4-Chloro-2-methylaniline & $95-69-2$ & 0.35 & 0.18 & \\
\hline 278 & 2-Methoxy-4-propenylphenol & $97-54-1$ & 0.75 & & 0.69 \\
\hline 279 & 2,4,6-tris(Dimethylaminomethyl)phenol & $90-72-2$ & -0.52 & $*$ & \\
\hline 280 & 2-Fluoroaniline & $348-54-9$ & -0.37 & 0.01 & \\
\hline 281 & 4-Aminobenzyl cyanide & $3544-25-0$ & -0.76 & -0.4 & \\
\hline 282 & 3-Iodoaniline & $626-01-7$ & 0.65 & & 0.44 \\
\hline 283 & 3-Cinnamonitrile & $4360-47-8$ & 0.16 & & 0.52 \\
\hline 284 & 3-Fluorobenzyl alcohol & $456-47-3$ & -0.39 & -0.16 & \\
\hline 285 & 3-Cyanoaniline & $2237-30-1$ & -0.47 & -0.24 & \\
\hline 286 & 4-Fluorophenol & $371-41-5$ & 0.02 & -0.00 & \\
\hline 287 & 2-Iodoaniline & $615-43-0$ & 0.35 & 0.49 & \\
\hline 288 & 3-Fluoroaniline & $372-19-0$ & -0.10 & 0.02 & \\
\hline 289 & 4-Chloro-2-methylphenol & $1570-64-5$ & 0.7 & 0.56 & \\
\hline 290 & 4-Chloro-3-ethylphenol & $14143-32-9$ & 1.08 & & 0.72 \\
\hline 291 & 2-Chloro-4,5-dimethylphenol & $1124-04-5$ & 0.69 & 0.96 & \\
\hline 292 & 3,5-Dimethoxyphenol & $500-99-2$ & -0.09 & -0.04 & \\
\hline 293 & 4-Hydroxybenzyl cyanide & 14191-95-8 & -0.38 & -0.23 & \\
\hline 294 & 4-Bromo-2,6-dimethylphenol & $2374-05-2$ & 1.16 & & 0.70 \\
\hline 295 & 2-Bromobenzyl alcohol & $18982-54-2$ & 0.10 & & 0.07 \\
\hline 296 & 2-Chloro-5-methylphenol & $615-74-7$ & 0.54 & 0.56 & \\
\hline 297 & 2-Fluorophenol & $367-12-4$ & 0.19 & -0.06 & \\
\hline 298 & 4-(Dimethylamino)benzaldehyde & $100-10-7$ & 0.23 & 0.15 & \\
\hline 299 & 4-Bromophenol & $106-41-2$ & 0.68 & 0.50 & \\
\hline 300 & 3-Chloro-2-methylaniline & $95-79-4$ & 0.50 & 0.14 & \\
\hline 301 & 3-Chloro-4-methylaniline & $95-74-9$ & 0.39 & & 0.19 \\
\hline 302 & 3-Chloro-2-methylaniline & $87-60-5$ & 0.38 & 0.14 & \\
\hline 303 & 4-Chlorophenethyl alcohol & $1875-88-3$ & 0.32 & 0.12 & \\
\hline 304 & 4-Chlorobenzyl alcohol & $873-76-7$ & 0.25 & 0.10 & \\
\hline 305 & 2-Bromo-4-methylphenol & $6627-55-0$ & 0.60 & 0.57 & \\
\hline 306 & 1,3,5-Trimethyl-2-nitrobenzene & $603-71-4$ & 0.86 & 1.08 & \\
\hline 307 & 3-Chlorobenzyl alcohol & $873-63-2$ & 0.15 & 0.10 & \\
\hline 308 & 2-Bromophenol & $95-56-7$ & 0.33 & & 0.51 \\
\hline 309 & 4-Hydroxy-3-methoxybenzonitrile & $4421-08-3$ & -0.03 & -0.14 & \\
\hline 310 & 3-Nitrobenzyl alcohol & $619-25-0$ & -0.22 & & 0.25 \\
\hline 311 & 4-Bromophenyl acetonitrile & $16532-79-9$ & 0.6 & 0.26 & \\
\hline 312 & 4-Methoxybenzonitrile & $874-90-8$ & 0.10 & -0.14 & \\
\hline 313 & 2-Hydroxy-4,5-dimethylacetophenone & $36436-65-4$ & 0.71 & & 0.57 \\
\hline 314 & 2-Anisaldehyde & $135-02-4$ & 0.15 & & -0.03 \\
\hline 315 & 4-Chlororesorcinol & $95-88-5$ & 0.13 & 0.59 & \\
\hline 316 & Methyl-4-methylaminobenzoate & $18358-63-9$ & 0.31 & 0.19 & \\
\hline 317 & 4-Phenoxybenzaldehyde & $67-36-7$ & 1.26 & 1.26 & \\
\hline 318 & 3-Hydroxy-4-methoxybenzaldehyde & $621-59-0$ & -0.14 & -0.08 & \\
\hline 319 & 4-Biphenylcarboxaldehyde & $3218-36-8$ & 1.12 & 0.95 & \\
\hline 320 & 2,4,5-Trimethoxybenzaldehyde & $4460-86-0$ & -0.10 & 0.36 & \\
\hline 321 & 4-Benzoylaniline & $1137-41-3$ & 0.68 & 0.94 & \\
\hline 322 & 3-Anisaldehyde & $5991-31-1$ & 0.23 & -0.08 & \\
\hline 323 & $n$-Propyl cinnamate & $7778-83-8$ & 1.23 & 1.12 & \\
\hline 324 & (trans)-Ethyl cinnamate & $103-36-6$ & 0.99 & 0.71 & \\
\hline 325 & Hexanophenone & $942-92-7$ & 1.19 & 1.11 & \\
\hline 326 & $n$-Butyl cinnamate & $538-65-8$ & 1.53 & 1.75 & \\
\hline 327 & 4-Chlorobenzyl cyanide & $140-53-4$ & 0.66 & 0.21 & \\
\hline 328 & (trans)-Methyl cinnamate & $103-26-4$ & 0.58 & 0.38 & \\
\hline
\end{tabular}




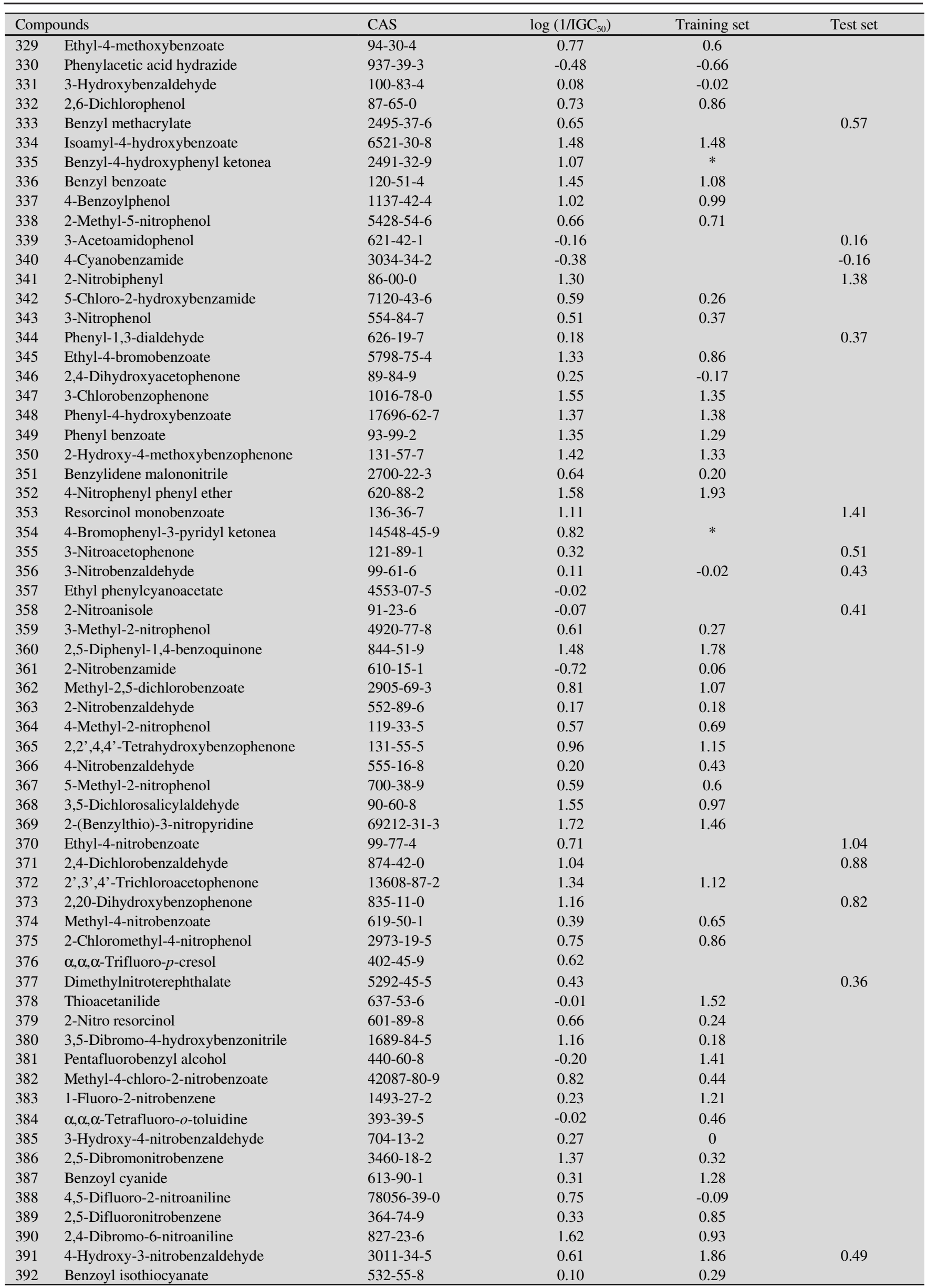


gas-phase geometry calculation of AM1. Twenty three constant and near constant variables exclude from descriptors and then 193 descriptors have low correlations $(<0.1)$ with $\log \left(\mathrm{IGC}_{50}\right)$ and 677 descriptors with pair correlations 0.98 exclude. The total remaining descriptors were 134 and we use these descriptors for stepwise variable selection.

Cluster analysis: The cluster analysis (CA) is the name of a group of methods used to recognize similarities among cases (objects) or among variables and to single out some categories as a set of similar cases (or variables) ${ }^{13}$. This cluster analysis comprehends a number of different 'classification algorithms' and it allows organizing the data into subsystems. These algorithms are grouped into two categories: hierarchical clustering and partitional (non-hierarchical) clustering. Hierarchical clustering rearranges objects in a tree structure (joining clustering) in an agglomerative (bottom-up) procedure. On the other hand, partitional clustering assumes that the objects have non-hierarchical characters ${ }^{14}$. The most used cluster algorithms are the k-means cluster analysis (k-MCA) and Jarvis-Patrick algorithm (also known as k-nearest neighbor cluster analysis; k-NNCA); in our case, in order to design the training and test series to guarantee structural and toxicity variability in both series of the present database, we carried out k-MCA for the entire dataset of compounds. This approach (clustering) ensures that the similarity principle can be employed for the activity prediction of the test set. The number of members in each cluster and the standard deviation of the variables in the cluster (kept as low as possible) were taking into account, to have an acceptable statistical quality of data partition into clusters. The values of the standard deviation between and within clusters, those of the respective Fisherratio and their $p$-level of significance were also examined ${ }^{14,15}$. Finally, before carrying out the cluster processes, all the variables were standardized. In standardization, all values of selected variables (molecular descriptors) were replaced by standardized values, which are computed as follows:

$$
\mathrm{Z}=(\mathrm{x}-\mathrm{mu}) / \sigma
$$

where, $\mathrm{Z}$ and $\mathrm{x}$ are normal distribution of $\mathrm{z}$-scores and each raw score, mu and $\sigma$ are normal mean and standard deviation of a set of scores.

Stepwise multiple linear regression modeling: The general purpose of multiple regressions is to quantify the relationship between several independent or predictor variables and a dependent variable. A set of coefficients defines the single linear combination of independent variables (molecular descriptors) that best describes compound $\log \left(\mathrm{IGC}_{50}\right)$. The $\log \left(\mathrm{IGC}_{50}\right)$ value for each benzene derivative would then be calculated as a composite of each molecular descriptor weighted by the respective coefficients. A multilinear model can be represented as:

$$
y=\beta_{0}+\beta_{1} X_{1}+\beta_{2} X_{2}+\beta_{3} x_{3}+\cdots+\beta_{k} X_{k}
$$

where, $\mathrm{k}$ is the number of independent variables, $\beta_{1} \ldots \beta_{\mathrm{k}}$, the regression coefficients and $y$ is the dependent variable. Regression coefficients represent the independent contributions of each calculated molecular descriptor.

A single multiple linear regression model was developed for benzene derivative compounds using the SPSS version 11.5 software. The multiple linear regression model was built using a training set and validation using an external prediction set.
Multiple linear regression techniques based on least-squares procedures are very often used for estimating the coefficients involved in the model equation ${ }^{16}$.

The stepwise multiple linear regression is a commonly used variant of multiple linear regression. In this case, also a multiple-term linear equation is produced, but not all independent variables are used. Each variable is added to the equation at a time and a new regression is performed. The new term is retained only if equation passes a test for significance. This regression method is especially useful when the number of variables is large and when the key descriptors are not known. Usually, molecular descriptor matrices cannot be directly used as independent variables in the multiple linear regression analysis due to their lack of homogeneity, the high correlation between descriptors is larger than the number of compounds, some of them maybe redundant. Thus previous to the multiple linear regression analysis, normally a reduction of variables is necessary in order to obtain a concentrated set of significant underlying variables, not correlated between them, losing the minimum amount of information.

Partial least square: Partial least square is a generalization of regression, which can handle data with strongly correlated and/or noisy or numerous $X$ variables ${ }^{17}$. It gives a reduced solution, which is statistically more robust than multiple linear regression. The linear partial least square model finds new variables (latent variables or X scores), which are linear combinations of the original variables. The latent variables in partial least square are also linear combinations of the descriptive variables in the data set, but instead of maximizing the variance in the matrix with descriptive variables like in principle component analysis (PCA), the covariance with the response variable is maximized. The scores on the partial least square factors are used as input for multiple linear regression after selection of the optimal number of partial least square-factors to be considered ${ }^{18}$.

To avoid overfitting, a strict test for the significance of each consecutive partial least square component is necessary and then stopping when the components are non-significant. Cross-validation is a reliable and commonly used method for testing this significance ${ }^{19}$. However, recently it has been shown that from the viewpoint of external predictability, choice of variables for partial least square based on internal validation may not be optimum ${ }^{15}$. Application of partial least square allows the construction of larger QSAR equations while still avoiding overfitting and eliminating most variables. Partial least square is normally used in combination with crossvalidation to obtain the optimum number of components. This ensures that the QSAR equations are selected based on their ability to predict the data rather than to fit the data ${ }^{16}$. Based on the standardized regression coefficients, the variables with smaller coefficients were removed from the partial least square regression, until there was no further improvement in $\mathrm{Q}^{2}$ value, irrespective of the components.

\section{RESULTS AND DISCUSSION}

Similarity analysis and design of training and test sets: Principle component analysis study of 392 benzene derivatives X-matrix data, showed compounds 153, 172, 279, 335 and 354 (3,4,5,6-tetrabromo-o-cresol, pentabromophenol, 2,4,6- 
tris(dimethylaminomethyl) phenol benzyl-4-hydroxyphenyl ketone and 4-bromophenyl-3-pyridyl) are as statistical outlieres, once rejected the statistical outliers, in order to split the whole group into two datasets (training and predicting ones), we perform a k-MCA. The main idea of this procedure consists in making a partition of chemicals in several statistically representative classes of compounds. This procedure ensures that any chemical class (as determined by the clusters derived from k-MCA) will be represented in both compounds' series. This rational design of the training and predicting series allowed us to design both sets: that are representative of the whole experimental universe. This procedure split the dataset of benzene derivatives into 9 clusters. Afterward, the selection of the training and prediction sets was performed by taking, in a random way, compounds belonging to each cluster. From these 387 compounds, 309 were chosen at random to form the training set. The remaining subset, composed of 78 compounds, was prepared as test set for the external set validation of the models. These compounds were never used in the development of the classification models. Fig. 1 illustrates graphically the above-described procedure, where a cluster analyses was performed to select a representative sample for the training and test sets.

Stepwise multiple linear regression and partial least square: Partial least square was applied to the data set after selection of descriptors by the stepwise multiple linear regression. The stepwise multiple linear regression algorithm was applied to the data set using the decimal logarithm as the log $\left(\mathrm{IGC}_{50}\right)$ values as response variables and the autoscaled calculated descriptors as independent variables. For evaluation of the predictive power of the generated partial least square, the optimized model was applied for prediction of $\log \left(\mathrm{IGC}_{50}\right)$ values of 78 compounds in the prediction set, which were not used in the optimization procedure. Table- 2 show the selected

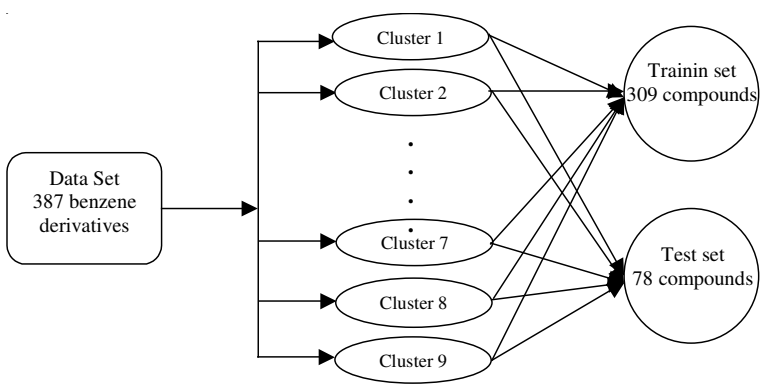

Fig. 1. General algorithm used for designing training and test sets throughout k-MCA

descriptors, their definition and class partial least square regression applied in the modeling procedure (stepwise MLRPLS). For the constructed model, the predictive ability of the partial least square model was evaluated by calculation of statistical parameters. The root mean square error of calibration and prediction (RMSEC and RMSEP), predicted residual error sum of square (PRESS), standard error of prediction (SEP), the percent relative errors ( $\% \mathrm{RE})$, square adjusted correlation coefficient for the training and prediction set $\left(\mathrm{R}^{2}\right.$ and $\left.\mathrm{R}_{\text {pred }}\right)$ obtained by the partial least square method are presented in Table-3. The coefficient of determination equals 0.8104 and the RMSEC value is 0.3296 . These values show that the stepwise MLR-PLS model fits the calibration data well and also has good predictive abilities $\left(\mathrm{R}_{\text {Pred }}^{2}=0.819\right)$. The residual plot (Fig. 2) of the stepwise MLR-PLS model shows considerable small residuals. The plot of predicted $\log \left(\mathrm{IGC}_{50}\right)$ versus experimental $\log \left(\mathrm{IGC}_{50}\right)$ obtained by the stepwise MLR-PLS modeling is shown in Fig. 3. Also the agreement observed between the predicted and experimental $\log \left(\mathrm{IGC}_{50}\right)$ values in Fig. 4 confirms a good predictive ability of stepwise MLRPLS modeling. The partial least square latent variable for this data by this model was obtained ${ }^{\text {. }}$.

TABLE-2

SELECTED DESCRIPTORS IN THE STEPWISE MLR MODEL AND STANDARDIZED COEFFICIENT FOR STEPWISE MLR-PLS MODEL

\begin{tabular}{lllc}
\hline Descriptor & Definition & Descriptor class & $\begin{array}{c}\text { Standardized } \\
\text { coefficient (stepwise } \\
\text { MLR-PLS model) }\end{array}$ \\
\hline MLOGP & Moriguchi octanol-water partition coefficient (logP) & 0.2232 \\
RARS & R matrix average row sum & Molecular properties & -0.1846 \\
AM & A total size index/weighted by atomic masses & GETAWAY descriptors & 0.2852 \\
H-046 & H attached to C0 $\left(\right.$ sp $^{3}$ ) no X attached to next C & WHIM descriptors & 0.3278 \\
BELM1 & Lowest eigenvalue n. 1 of Burden matrix/weighted by atomic masses & Atom-centered fragments & Burden eigen values \\
PCWTE & Partial charge weighted topological electronic descriptor & Charge descriptors & -0.2294 \\
RDF040M & Radial Distribution Function - 4.0/ weighted by atomic masses & RDF descriptors & 0.1498 \\
FDI & Folding degree index & Geometrical descriptors & 0.2372 \\
MOR10P & 3D-MoRSE -signal 01/weighted by atomic polarizabilities & 3D-MoRSE descriptors & 0.0964 \\
MOR06U & 3D-MoRSE -signal 06/unweighted & 3D-MoRSE descriptors & 0.0556 \\
SHP2 & Average shape profile index of order 2 & Randic molecular profiles & -0.2570 \\
JGI3 & Mean topological charge index of order3 & Topological charge indices & 0.2274 \\
RDF050M & Radial Distribution Function - 5.0/weighted by atomic masses & RDF descriptors & -0.2120 \\
MOR18M & 3D-MoRSE -signal 18/weighted by atomic masses & 3D-MoRSE descriptors & 0.1470 \\
RDF010M & Radial Distribution Function-1.0/weighted by atomic masses & RDF descriptors & -0.0976 \\
MOR05M & 3D-MoRSE -signal 05/weighted by atomic masses & 3D-MoRSE descriptors & -0.1406 \\
\hline
\end{tabular}

TABLE-3

OVERVIEW OF STEPWISE MLR-PLS MODEL

\begin{tabular}{lccccccc}
\hline Method & RMSEC & RMSEP & REP $(\%)$ & SEP & PRESS & R $^{2}$ (adjusted) & $\mathrm{R}_{\text {Pred }}^{2}$ (adjusted) \\
\hline Stepwise MLR-PLS & 0.32 & 0.29 & 50.72 & 0.29 & 6.55 & 0.80 & 0.81 \\
\hline
\end{tabular}




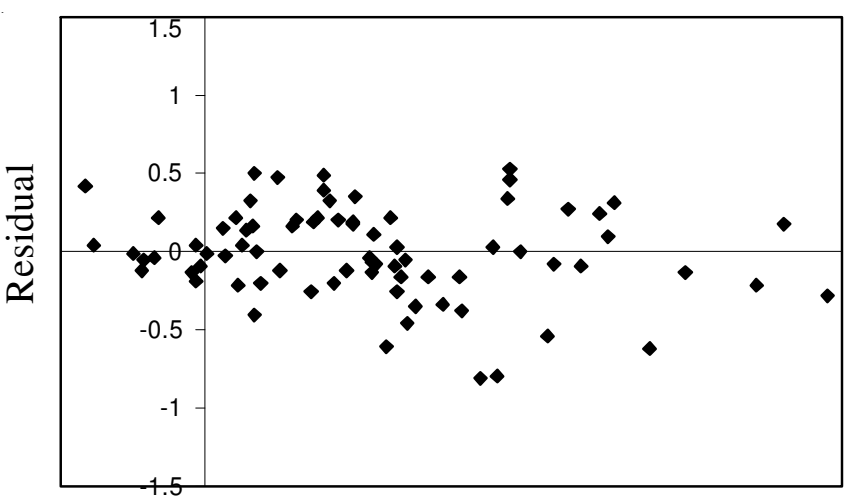

Predicted $\log \left(\mathrm{IGC}_{50}\right)$

Fig. 2. Residual plot for the stepwise MLR-PLS model

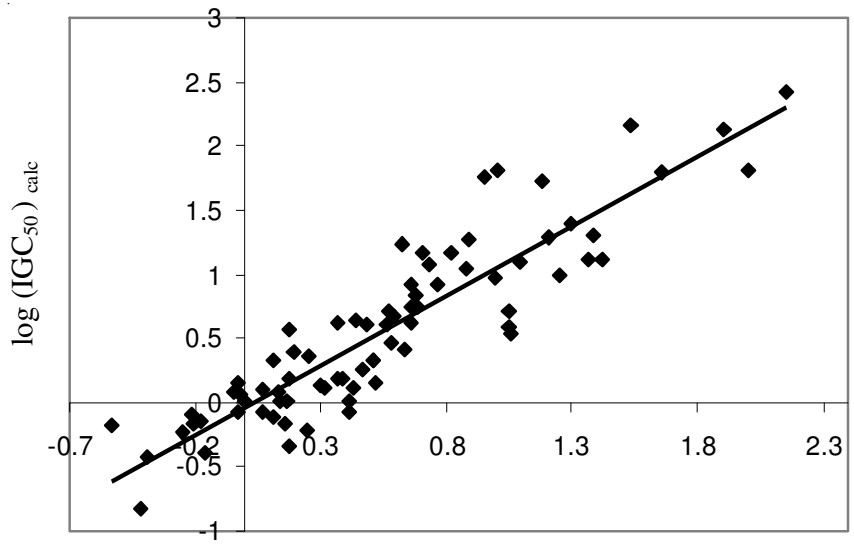

$\log \left(\mathrm{IGC}_{50}\right)$ exp

Fig. 3. Predicted $\log \left(\mathrm{IGC}_{50}\right)$ by stepwise MLR-PLS modeling versus experimental $\log \left(\mathrm{IGC}_{50}\right)$ for test molecules in prediction

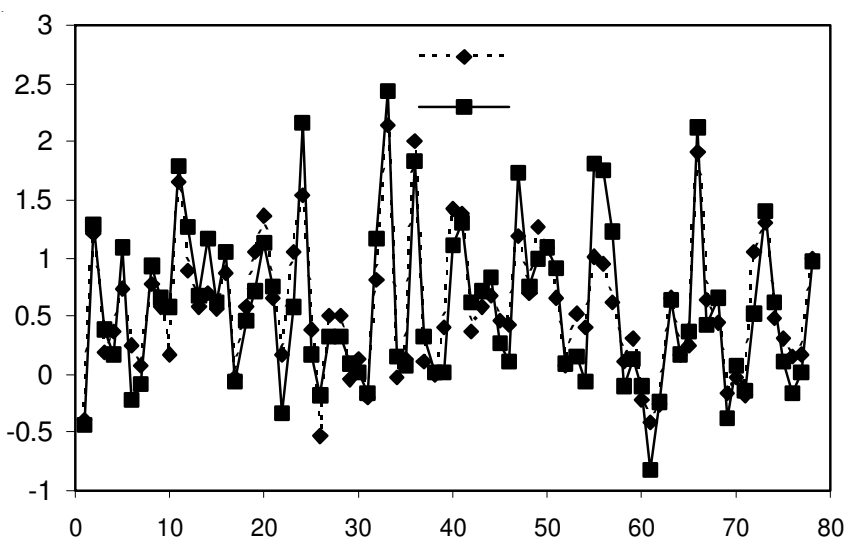

Fig. 4. Plots of experimental and predicted $\log \left(\mathrm{IGC}_{50}\right)$ values by stepwise MLR-PLS modeling versus sample number in the prediction set
The descriptor $\mathrm{H}-046$ is based on number of hydrogen attached to $\mathrm{CO}\left(s p^{3}\right)$ (carbon atom with formal oxidation number 0 and hybridization $\left.s p^{3}\right)$ with $\mathrm{X}(\mathrm{O}, \mathrm{N}, \mathrm{S}, \mathrm{P}, \mathrm{Se}$ or halogens) attached to next $\mathrm{C}$. The MLOGP descriptor is a measure for the Moriguchi octanol-water partition coefficient (log P). Most of the other descriptors can be related to the two-dimensional (BELM1) or three-dimensional (RARS, AM, PCWTE, RDF040M). Positive values in the regression coefficients indicate that the indicated descriptor contributes positively to the value of $\log$ $\left(\mathrm{IGC}_{50}\right)$, whereas negative values indicate that the greater the value of the descriptor the lower the value of $\log \left(\mathrm{IGC}_{50}\right)$.

Our method favourably compares with other approaches implemented in the Dragon software and atom-based nonstochastic and stochastic linear indices for this data set ${ }^{20}$. All these results are summarized in Table-4, where a detailed comparison can be more easily performed. The results were obtained on different sets of molecules, since they are based on different test sets. As it can be seen, our model has statistical parameter better than models obtained with reference ${ }^{20}$. In this sense, the present approach showed the greater values of squared adjusted correlation coefficient of $0.8104\left(\mathrm{R}^{2}{ }_{\text {Pred }}=\right.$ 0.8195), with stepwise MLR-PLS, correspondingly.

Statistical parameters: For evaluation of the predictive power of the generated partial least square, the optimized model was applied for prediction of $\log \left(\mathrm{IGC}_{50}\right)$ values of 78 compounds in the prediction set which were not used in the optimization procedure. For the constructed model, five general statistical parameters were selected to evaluate the predictive ability of the model for $\log \left(\mathrm{IGC}_{50}\right)$ values. In this case, the predicted $\log \left(\mathrm{IGC}_{50}\right)$ 's of each sample in prediction step were compared with the experimental aquatic toxicity. The PRESS (predicted residual sum of squares) statistic appears to be the most important parameter accounting for a good estimate of the real predictive error of the models. Its small value indicates that the model predicts better than chance and can be considered statistically significant.

$$
\text { Pr ess }=\sum_{\mathrm{i}=1}^{\mathrm{n}}\left(\hat{\mathrm{y}}-\mathrm{y}_{\mathrm{i}}\right)^{2}
$$

Root mean square error of prediction or calibration (RMSEP or RMSEC) is a measurement of the average difference between predicted and experimental values, at the prediction step. Root mean square error of prediction can be interpreted as the average prediction error, expressed in the same units as the original response values. The RMSEP was obtained by the following formula:

STATISTICAL PARAMETERS OF THE QSAR MODELS OBTAINED USING DIFFERENT MOLECULAR DESCRIPTORS TO PREDICT AQUATIC TOXICITY ${ }^{17}$

\begin{tabular}{lll}
\hline Index & & $\mathrm{R}^{2}$ \\
\hline Non-stochastic linear indices & ${ }^{\mathrm{p}} \mathrm{f}_{0 \mathrm{~L}}^{\mathrm{H}}\left(\mathrm{x}_{\mathrm{E}}\right),{ }^{\mathrm{k}} \mathrm{f}_{0}(\mathrm{x}),{ }^{\mathrm{p}} \mathrm{f}_{1 \mathrm{~L}}^{\mathrm{H}}\left(\mathrm{x}_{\mathrm{E}}\right),{ }^{\mathrm{p}} \mathrm{f}_{3 \mathrm{~L}}\left(\mathrm{x}_{\mathrm{E}}\right),{ }^{\mathrm{pp}} \mathrm{f}_{3}(\mathrm{x}),{ }^{\mathrm{M}} \mathrm{f}_{9 \mathrm{~L}}^{\mathrm{H}}\left(\mathrm{x}_{\mathrm{E}}\right)$ & 0.721 \\
Stochastic linear indices & ${ }^{\mathrm{Ms}} \mathrm{f}_{15}(\mathrm{x}),{ }^{\mathrm{ks}} \mathrm{f}_{0}^{\mathrm{H}}(\mathrm{x}),{ }^{\mathrm{ks}} \mathrm{f}_{5}^{\mathrm{H}}(\mathrm{x}),{ }^{\mathrm{Gs}} \mathrm{f}_{0}^{\mathrm{H}}(\mathrm{x}),{ }^{\mathrm{Vs}} \mathrm{f}_{4 \mathrm{~L}}^{\mathrm{H}}\left(\mathrm{x}_{\mathrm{E}}\right),{ }^{\mathrm{Vs}} \mathrm{f}_{2 \mathrm{H}}^{\mathrm{H}}\left(\mathrm{x}_{\mathrm{E}}\right)$ & 0.733 \\
2D autocorrelations & $\mathrm{ATS} 3 \mathrm{v}$, ATS8v, ATS3e, ATS8e, MATS1e, GATS1m & 0.609 \\
BCUT & BEHm7, BELm4, BELm6, BELv4, BELe6, BEHp6 & 0.690 \\
Gálvez topological charge indices & GGI2, GGI6, GGI8, JGI2, JGI5, JGI8 & 0.516 \\
Topological descriptors & ISIZ, X2sol, S2K,PW2, TIC1, pilD & 0.716 \\
Molecular walk count & MWC08, MWC09, TWC, SRW10 & 0.346 \\
\hline
\end{tabular}




$$
\operatorname{RMSEP}(\text { or RMSEC })=\left[\frac{1}{\mathrm{n}} \sum_{\mathrm{i}=1}^{\mathrm{n}}\left(\hat{\mathrm{y}}-\mathrm{y}_{\mathrm{i}}\right)^{2}\right]^{0.5}
$$

The third statistical parameter was relative error of prediction (REP) that shows the predictive ability of each component and is calculated as:

$$
\operatorname{REP}(\%)=\frac{100}{\mathrm{n}}\left[\frac{1}{\mathrm{n}} \sum_{\mathrm{i}=1}^{\mathrm{n}}\left(\hat{\mathrm{y}}-\mathrm{y}_{\mathrm{i}}\right)^{2}\right]^{0.5}
$$

The predictive applicability of a regression model is described in various ways. The most general expression is the standard error of prediction (SEP) which is given in the following formula:

$$
\mathrm{SEP}=\left[\frac{\sum_{\mathrm{i}=1}^{\mathrm{n}}\left(\hat{\mathrm{y}}_{\mathrm{i}}-\mathrm{y}_{\mathrm{i}}\right)^{2}}{\mathrm{n}-1}\right]^{0.5}
$$

The square of the adjusted correlation coefficient, which is, indicated the quality of fit of all the data to a straight line is calculated for the checking of test set and is calculated as:

$$
\mathrm{R}_{\text {Pred }}^{2}=1-\frac{\sum_{\mathrm{i}=1}^{\mathrm{n}}\left(\mathrm{y}_{\mathrm{i}}-\hat{\mathrm{y}}_{\mathrm{i}}\right)^{2}}{\sum_{\mathrm{i}=1}^{\mathrm{n}}\left(\mathrm{y}_{\mathrm{i}}-\overline{\mathrm{y}}\right)^{2}}
$$

where, $\mathrm{y}_{\mathrm{i}}$ is the experimental $\log \left(\mathrm{IGC}_{50}\right)$ of the benzene derivatives in the sample $\mathrm{i}, \hat{\mathrm{y}}_{\mathrm{i}}$ represented the predicted $\log$ $\left(\mathrm{IGC}_{50}\right)$ of the benzene derivatives in the sample $\mathrm{i}, \overline{\mathrm{y}}$, is the mean of experimental $\log \left(\mathrm{IGC}_{50}\right)$ in the prediction set and $\mathrm{n}$ is the total number of samples used in the prediction set. The statistical results (PRESS, RMSEC, RMSEP, REP \%, SEP and $\mathrm{R}^{2}$ ) are summarized in Table-3.

\section{Conclusion}

We have developed here a useful QSAR equation derived from quantum chemical descriptors associated with aquatic toxicity properties of 392 benzene derivatives. For each compound 134 descriptors, 11 classes of Dragon descriptors, calculated. The dataset was carefully split into training and test sets, guaranteeing enough molecular diversity in each subset, by using k-MCA cluster analysis. Then the best set of calculated descriptors was selected by stepwise multiple linear regression. Model was obtained with partial least square regression. Stepwise MLR-PLS is successfully presented for prediction aquatic toxicity $\left[\log \left(1 / \mathrm{IGC}_{50}\right)\right]$ of various benzene derivatives $\left(R_{\text {Pred }}^{2}=0.8195\right)$ with diverse chemical structures using a linear quantitative structure-activity property relationship. This model with high statistical quality and low prediction errors was obtained. In general, it can be concluded that, for this data set, the combinations of linear modeling techniques result in an improvement of the linear models. The results indicate that four descriptors, Moriguchi octanol-water partition coefficient $(\log \mathrm{P})(\mathrm{M} \log \mathrm{P}), \mathrm{H}$ attached to $\mathrm{C}_{0}\left(s p^{3}\right)$ number $\mathrm{X}$ attached to next $\mathrm{C}$ (H-046), A total size index/ weighted by atomic masses (AM) and R matrix average row sum (RARS) were selected and play an important role on the aquatic toxicity of benzene derivatives structure.

Development of quantitative structure-property/activity relationships (QSPR/QSAR) on theoretical descriptors is a powerful tool not only for prediction of the chemical, physical and biological properties/activities of compounds, but also for deeper understanding of the detailed mechanisms of aquatic toxicity in benzene derivatives that predetermine these activity.

\section{REFERENCES}

1. A. Worth, A. Bassan, J. De Bruijn, A. Saliner, T. Netzeva, G. Patlewicz, M. Pavan, I. Tsakovska and S. Eisenreich, SAR QSAR Environ. Res., 18, 111 (2007).

2. C. Hansch, P.P. Maloney, T. Fujita and R.M. Muir, Nature, 194, 178 (1962).

3. R. Todeschini and V. Consonni, Handbook of Molecular Descriptors, Weinheim: Wiley-VCH (2000).

4. M. Karelson, Molecular Descriptors in QSAR/QSPR, Wiley-Interscience (2000); B. Hemmateenejad, M. Safarpour and F. Taghavi, J. Mol. Struct. (Theochem.), 635, 183 (2003); I. Moriguchi, S. Hirono, Q. Liu, I. Nakagome and Y. Matsushita, Chem. Pharm. Bull. (Tokyo), 40, 127 (1992).

5. W. Tong, H. Hong, Q. Xie, L. Shi, H. Fang and R. Perkins, Curr. Comput. Aided Drug. Des., 1, 195 (2005); L. He and P. Jurs, J. Mol. Graphics Model., 23, 503 (2005); T. Ghafourian and M. Cronin, SAR OSAR Environ. Res., 16, 171 (2005); A. Tropsha, P. Gramatica and V. Gombar, QSAR Comb. Sci., 22, 69 (2003); A. Golbraikh and A. Tropsha, J. Mol. Graphics Model., 20, 269 (2002)

6. S. Wold and L. Eriksson, Chemometric Methods in Molecular Design, VCH, Weinheim, Vol. 195 (1995).

7. S.D. Brown, S.T. Sum, F. Despagne and B.K. Lavine, Ana. Ch., 68, 21 (1996); K. Roy and J. Leonard, Bioorg. Med. Chem., 13, 2967 (2005).

8. T. Schultz, T. Netzeva, in Predicting Chemical Toxicity and Fate, p. 265 (2004).

9. S. Bradbury, C. Russom, G. Ankley, T. Schultz and J. Walker, Environ. Toxicol. Chem., 22, 1789 (2009).

10. R. Johnson and D. Wichern, Applied Multivariate Statistical Analysis, Prentice Hall Englewood Cliffs, NJ, (1998); J. Mc Farland and D. Gans, Chapter Cluster Significance Analysis, VCH, Weinheim, 295 (1995).

11. P. Broto, G. Moreau and C. Vandycke, Eur. J. Med. Chem., 19, 61 (1984); J. Gálvez, R. García, M. Salabert and R. Soler, J. Chem. Inf. Comput. Sci., 34, 520 (1994); L. Kier and L. Hall, Molecular Connectivity in Structure-Activity Analysis, Wiley, New York (1986); E. Konstantinova, J. Chem. Inf. Comput. Sci., 36, 54 (1996); G. Ruecker and C. Ruecker, J. Chem. Inf. Comput. Sci., 33, 683 (1993).

12. M. Dewar, E. Zoebisch, E. Healy and J. Stewart, J. Am. Chem. Soc., 107, 3902 (1985)

13. J. Xu and A. Hagler, Molecules, 7, 566 (2002).

14. R. Leardi and A. Lupiáñez González, Chemometrics Intell. Lab. Syst., 41, 195 (1998).

15. P. Geladi and B. Kowalski, Anal. Chim. Acta, 185, 1 (1986).

16. R. Leardi, R. Boggia and M. Terrile, J. Chemometrics, 6, 267 (2005).

17. D. Goldberg, Genetic Algorithms in Search, Optimization and Machine Learning Addision Wesley Longman Inc, MA, USA (1989).

18. R. Yu, Introduction to Chemometrics, Changsha, Hunan Education House, Vol. 67 (1991).

19. B. Dayal and J. MacGregor, J. Chemometrics, 11, 73 (1998).

20. J. Castillo-Garit, Y. Marrero-Ponce, J. Escobar, F. Torrens and R. Rotondo, Chemosphere, 73, 415 (2008). 